\title{
Generalized statistical convergence of difference sequences
}

\author{
Abdullah Alotaibi ${ }^{1}$ and Mohammad Mursaleen ${ }^{2 *}$
}

\section{"Correspondence:}

mursaleenm@gmail.com

${ }^{2}$ Department of Mathematics,

Aligarh Muslim University, Aligarh,

202002, India

Full list of author information is

available at the end of the article

\begin{abstract}
In this paper we define the $\lambda(u)$-statistical convergence that generalizes, in a certain sense, the notion of $\lambda$-statistical convergence. We find some relations with sets of sequences which are related to the notion of strong convergence.
\end{abstract}

MSC: 40A05; 40H05

Keywords: statistical convergence; $\lambda$-statistical convergence; difference sequences

\section{Introduction and preliminaries}

The notion of statistical convergence (see Fast [1]) has been studied in various setups, and its various generalizations, extensions and variants have been studied by various authors so far. For example, lacunary statistical convergence [2], $A$-statistical convergence [3, 4], statistical summability $(C, 1)[5,6]$, statistical $\lambda$-summability [7], statistical $\sigma$-convergence [8], statistical $A$-summability [9], $\lambda$-statistical convergence with order $\alpha$ [10], lacunary and $\lambda$-statistical convergence in a solid Riesz space $[11,12]$, lacunary statistical convergence and ideal convergence in random 2-normed spaces $[13,14]$, generalized weighted statistical convergence [15] etc. In this paper we define the notion of $\lambda$-statistical convergence as a matrix domain of a difference operator [16], which is obtained by replacing the sequence $x$ by $u \Delta x$, where $\Delta x=\left(x_{k}-x_{k+1}\right)_{k=1}^{\infty}$ and $u=\left(u_{k}\right)_{k=1}^{\infty}$ is another sequence with $u_{k} \neq 0$ for all $k$. We find some relations with sets of sequences which are related to the notion of strong convergence [17].

Let $K$ be a subset of the set of natural numbers $\mathbb{N}$. Then the asymptotic density of $K$ denoted by $\delta(K)$ is defined as $\delta(K)=\lim _{n} \frac{1}{n}|\{k \leq n: k \in K\}|$, where the vertical bars denote the cardinality of the enclosed set.

A number sequence $x=\left(x_{k}\right)$ is said to be statistically convergent to the number $L$ if for each $\epsilon>0$, the set $K(\epsilon)=\left\{k \leq n:\left|x_{k}-L\right|>\epsilon\right\}$ has asymptotic density zero, i.e.,

$$
\lim _{n} \frac{1}{n}\left|\left\{k \leq n:\left|x_{k}-L\right|\right\}\right|=0
$$

In this case, we write $S-\lim x=L$.

Let $\lambda=\left(\lambda_{n}\right)$ be a non-decreasing sequence of positive numbers tending to $\infty$ such that

$$
\lambda_{n+1} \leq \lambda_{n}+1, \quad \lambda_{1}=0 .
$$

2013 Alotaibi and Mursaleen; licensee Springer. This is an Open Access article distributed under the terms of the Creative Commons Attribution License (http://creativecommons.org/licenses/by/2.0), which permits unrestricted use, distribution, and reproduction in any medium, provided the original work is properly cited. 
The generalized de la Vallée-Poussin mean is defined by

$$
t_{n}(x)=: \frac{1}{\lambda_{n}} \sum_{j \in I_{n}} x_{j}
$$

where $I_{n}=\left[n-\lambda_{n}+1, n\right]$.

A sequence $x=\left(x_{j}\right)$ is said to be $(V, \lambda)$-summable to a number $L$ if

$$
t_{n}(x) \rightarrow L \quad \text { as } n \rightarrow \infty
$$

In this case, $L$ is called the $\lambda$-limit of $x$.

Let $K \subseteq \mathbb{N}$. Then the $\lambda$-density of $K$ is defined by

$$
\delta_{\lambda}(K)=\lim _{n} \frac{1}{\lambda_{n}}\left|\left\{n-\lambda_{n}+1 \leq j \leq n: j \in K\right\}\right| .
$$

In case $\lambda_{n}=n, \lambda$-density reduces to the asymptotic density. Also, since $\left(\lambda_{n} / n\right) \leq 1, \delta(K) \leq$ $\delta_{\lambda}(K)$ for every $K \subseteq \mathbb{N}$.

A sequence $x=\left(x_{k}\right)$ is said to be $\lambda$-statistically convergent (see [12]) to $L$ if for every $\epsilon>0$ the set $K_{\epsilon}:=\left\{k \in \mathbb{N}:\left|x_{k}-L\right| \geq \epsilon\right\}$ has $\lambda$-density zero, i.e., $\delta_{\lambda}\left(K_{\epsilon}\right)=0$. That is,

$$
\lim _{n} \frac{1}{\lambda_{n}}\left|\left\{k \in I_{n}:\left|x_{k}-L\right| \geq \epsilon\right\}\right|=0 .
$$

In this case, we write $S_{\lambda}-\lim x=L$ and we denote the set of all $\lambda$-statistically convergent sequences by $S_{\lambda}$.

\section{$2 \lambda(u)$-Statistical convergence}

We consider the infinite matrix of first difference $\Delta=\left(a_{n m}\right)_{n, m \geq 1}$ defined by $a_{n n}=1, a_{n, n+1}=$ -1 and $a_{n m}=0$ otherwise. Let $D_{u}$ be the diagonal matrix defined by $\left[D_{u}\right]_{n n}=u_{n}$ for all $n$ and consider the set $U$ of all sequences such that $u_{n} \neq 0$ for all $n$. Then we write $\Delta(u)=D_{u} \Delta$ for $u \in U$.

From the generalized de la Vallée-Poussin mean defined by

$$
t_{n}(x)=\frac{1}{\lambda_{n}} \sum_{k \in I_{n}} x_{k} \quad \text { for } x=\left(x_{k}\right)_{k}
$$

we are led to define the following sets:

$$
\begin{aligned}
{[V, \lambda]_{0}(\Delta(u)) } & =\left\{x=\left(x_{k}\right): \lim _{n \rightarrow \infty} \frac{1}{\lambda_{n}} \sum_{k \in I_{n}}\left|\Delta(u) x_{k}\right|=0\right\} \\
& =\left\{x=\left(x_{k}\right): \lim _{n \rightarrow \infty} \frac{1}{\lambda_{n}} \sum_{k \in I_{n}}\left|u_{k}\left(x_{k}-x_{k+1}\right)\right|=0\right\}, \\
{[V, \lambda]_{\infty}(\Delta(u)) } & =\left\{x=\left(x_{k}\right): \sup _{n} \frac{1}{\lambda_{n}} \sum_{k \in I_{n}}\left|\Delta(u) x_{k}\right|<\infty\right\} \\
& =\left\{x=\left(x_{k}\right): \sup _{n} \frac{1}{\lambda_{n}} \sum_{k \in I_{n}}\left|u_{k}\left(x_{k}-x_{k+1}\right)\right|=0\right\} .
\end{aligned}
$$


In the case when $\lambda_{n}=n$, we write the previous sets $[V]_{0}(\Delta(u))$ and $[V]_{\infty}(\Delta(u))$, respectively. Now we can state the definition of $\lambda(u)$-statistical convergence to zero.

A sequence $x=\left(x_{k}\right)_{k \geq 1}$ is said to be $\lambda(u)$-statistically convergent to zero if for every $\varepsilon>0$,

$$
\lim _{n \rightarrow \infty} \frac{1}{\lambda_{n}}\left|\left\{k \in I_{n}:\left|\Delta(u) x_{k}\right| \geq \varepsilon\right\}\right|=0 .
$$

In this case, we write $x_{k} \rightarrow 0 S_{\lambda}(\Delta(u))$. If $\lambda_{n}=n$ for all $n$, we then write $x_{k} \rightarrow 0 S(\Delta(u))$.

\section{Main results}

We are ready to prove the following result.

\section{Theorem 1 Let $u \in U$. Then}

(a) $[V, \lambda]_{0}(\Delta(u)) \subset S_{\lambda}^{0}(\Delta(u))$ and the inclusion is proper,

(b) if $x \in l_{\infty}$ and $x_{k} \rightarrow 0 S_{\lambda}(\Delta(u))$, then $x \in[V, \lambda]_{0}(\Delta(u))$,

(c) $S_{\lambda}^{0}(\Delta(u)) \cap l_{\infty}=[V, \lambda]_{0}(\Delta(u)) \cap l_{\infty}$.

Proof (a) Let $\varepsilon>0$ be given and $x \in[V, \lambda]_{0}(\Delta(u))$. Then we have

$$
\frac{1}{\lambda_{n}} \sum_{k \in I_{n}}\left|\Delta(u) x_{k}\right| \geq \frac{1}{\lambda_{n}} \sum_{\substack{k \in I_{n} \\\left|x_{k}-L\right| \geq \varepsilon}}\left|\Delta(u) x_{k}\right| \geq \frac{\varepsilon}{\lambda_{n}}\left|\left\{k \in I_{n}:\left|\Delta(u) x_{k}\right| \geq \varepsilon\right\}\right| .
$$

Therefore $x \in S_{\lambda}^{0}(\Delta(u))$. The following example shows that the inclusion is proper: Let $x=\left(x_{k}\right)$ be defined by

$$
x_{k}= \begin{cases}\sum_{j=k}^{\infty} j, & \text { for } n-\left[\sqrt{\lambda_{n}}\right]+1 \leq k \leq n, \\ 0, & \text { otherwise. }\end{cases}
$$

Then $x \notin l_{\infty}$ and for $0<\varepsilon \leq 1$,

$$
\frac{1}{\lambda_{n}}\left|\left\{k \in I_{n}:\left|\Delta(u) x_{k}\right| \geq \varepsilon\right\}\right|=\frac{\left[\sqrt{\lambda_{n}}\right]}{\lambda_{n}} \rightarrow 0 \quad(n \rightarrow \infty),
$$

i.e., $x \in S_{\lambda}^{0}(\Delta(u))$. But

$$
\frac{1}{\lambda_{n}} \sum_{k \in I_{n}}\left|\Delta(u) x_{k}\right| \nrightarrow 0 \quad(n \rightarrow \infty)
$$

i.e., $x \notin[V, \lambda]_{0}(\Delta(u))$.

(b) Let $x \in l_{\infty}$ and $x_{k} \rightarrow 0 S_{\lambda}(\Delta(u))$. Then $\left|\Delta(u) x_{k}\right| \leq M$ for all $k$, where $M>0$. For $\varepsilon>0$, we have

$$
\begin{aligned}
\frac{1}{\lambda_{n}} \sum_{k \in I_{n}}\left|\Delta(u) x_{k}\right| & =\frac{1}{\lambda_{n}} \sum_{\substack{k \in I_{n} \\
\left|x_{k}-L\right| \geq \epsilon}}\left|\Delta(u) x_{k}\right|+\frac{1}{\lambda_{n}} \sum_{\substack{k \in I_{n} \\
\left|x_{k}-L\right|<\epsilon}}\left|\Delta(u) x_{k}\right| \\
& \leq \frac{M}{\lambda_{n}}\left|\left\{k \in I_{n}:\left|\Delta(u) x_{k}\right| \geq \varepsilon\right\}\right|+\varepsilon .
\end{aligned}
$$

Hence, $x \in[V, \lambda]_{0}(\Delta(u))$. 
(c) This immediately follows from (a) and (b).

This completes the proof of the theorem.

Theorem $2 S^{0}(\Delta(u)) \subseteq S_{\lambda}^{0}(\Delta(u))$ if and only if

$$
\liminf _{n \rightarrow \infty} \frac{\lambda_{n}}{n}>0
$$

where by $x \in S^{0}(\Delta(u))\left(\right.$ or $\left.x \in S_{\lambda}^{0}(\Delta(u))\right)$ we mean $x_{k} \rightarrow 0 S(\Delta(u))\left(\right.$ or $\left.x_{k} \rightarrow 0 S_{\lambda}(\Delta(u))\right)$.

Proof For $\varepsilon>0$, we have

$$
\left\{k \in I_{n}:\left|\Delta(u) x_{k}\right| \geq \varepsilon\right\} \subset\left\{k \leq n:\left|\Delta(u) x_{k}\right| \geq \varepsilon\right\} .
$$

Therefore

$$
\begin{aligned}
\frac{1}{n}\left|\left\{k \leq n:\left|\Delta(u) x_{k}\right| \geq \varepsilon\right\}\right| & \geq \frac{1}{n}\left|\left\{k \in I_{n}:\left|\Delta(u) x_{k}\right| \geq \varepsilon\right\}\right| \\
& \geq \frac{\lambda_{n}}{n} \cdot \frac{1}{\lambda_{n}}\left|\left\{k \in I_{n}:\left|\Delta(u) x_{k}\right| \geq \varepsilon\right\}\right| .
\end{aligned}
$$

Taking the limit as $n \rightarrow \infty$ and using $(*)$, we get the inclusion.

Conversely, suppose that

$$
\liminf _{n \rightarrow \infty} \frac{\lambda_{n}}{n}=0
$$

Choose a subsequence $(n(j))_{j \geq 1}$ such that $\frac{\lambda_{n(j)}}{n(j)}<\frac{1}{j}$. Define a sequence $x=\left(x_{k}\right)_{k \geq 1}$ such that

$$
\Delta x_{k}= \begin{cases}1, & \text { for } k \in I_{n(j)}, j=1,2,3, \ldots \\ 0, & \text { otherwise }\end{cases}
$$

Then $\Delta x \in[C, 1]$ and hence, by Theorem 2.1 of [18], $x \in S^{0}(\Delta(u))$. On the other hand, $x \notin[V, \lambda]_{0}(\Delta(u))$ and Theorem $1(\mathrm{~b})$ implies that $x \notin S_{\lambda}^{0}(\Delta(u))$. Hence, $(*)$ is necessary.

This completes the proof of the theorem.

Presently, for the reverse inclusion, we have only one way condition.

Theorem 3 If $\lim \sup _{n}\left(n-\lambda_{n}\right)<\infty$, then $S_{\lambda}^{0}(\Delta(u)) \subseteq S^{0}(\Delta(u))$.

Proof Let $\lim \sup _{n}\left(n-\lambda_{n}\right)<\infty$. Then there exists $M>0$ such that $n-\lambda_{n} \leq M$ for all $n$. Since $\frac{1}{n} \leq \frac{1}{\lambda_{n}}$ and $\left\{1 \leq k \leq n:\left|\Delta(u) x_{k}\right| \geq \varepsilon\right\} \subseteq\left\{k \in I_{n}:\left|\Delta(u) x_{k}\right| \geq \varepsilon\right\} \cup\left\{1 \leq k \leq n-\lambda_{n}\right.$ : $\left.\left|\Delta(u) x_{k}\right| \geq \varepsilon\right\}$, we have

$$
\begin{aligned}
& \frac{1}{n}\left|\left\{1 \leq k \leq n:\left|\Delta(u) x_{k}\right| \geq \varepsilon\right\}\right| \\
& \quad \leq \frac{1}{\lambda_{n}}\left|\left\{1 \leq k \leq n:\left|\Delta(u) x_{k}\right| \geq \varepsilon\right\}\right|
\end{aligned}
$$




$$
\begin{aligned}
& \leq \frac{1}{\lambda_{n}}\left|\left\{k \in I_{n}:\left|\Delta(u) x_{k}\right| \geq \varepsilon\right\}\right|+\frac{1}{\lambda_{n}}\left|\left\{k \leq n-\lambda_{n}:\left|\Delta(u) x_{k}\right| \geq \varepsilon\right\}\right| \\
& \leq \frac{1}{\lambda_{n}}\left|\left\{k \in I_{n}:\left|\Delta(u) x_{k}\right| \geq \varepsilon\right\}\right|+\frac{M}{\lambda_{n}} .
\end{aligned}
$$

Now, taking the limit as $n \rightarrow \infty$, we get $S_{\lambda}^{0}(\Delta(u)) \subseteq S^{0}(\Delta(u))$.

This completes the proof of the theorem.

\section{Competing interests}

The authors declare that they have no competing interests.

\section{Authors' contributions}

Both authors contributed equally and significantly in writing this paper. Both authors read and approved the final manuscript.

\section{Author details}

'Department of Mathematics, King Abdulaziz University, P.O. Box 80203, Jeddah, 21589, Saudi Arabia. ${ }^{2}$ Department of Mathematics, Aligarh Muslim University, Aligarh, 202002, India.

\section{Acknowledgements}

This project was funded by the Deanship of Scientific Research (DSR), King Abdulaziz University, Jeddah under Grant No. (174/130/1433). The authors, therefore, acknowledge with thanks DSR technical and financial support.

Received: 16 March 2013 Accepted: 26 June 2013 Published: 13 July 2013

\section{References}

1. Fast, H: Sur la convergence statistique. Colloq. Math. 2, 241-244 (1951)

2. Fridy, JA, Orhan, C: Lacunary statistical convergence. Pac. J. Math. 160, 43-51 (1993)

3. Alotaibi, A, Mursaleen, M: A-Statistical summability of Fourier series and Walsh-Fourier series. Appl. Math. Inf. Sci. 6(3), 535-538 (2012)

4. Kolk, E: The statistical convergence in Banach spaces. Tartu Ülik. Toim. 928, 41-52 (1991)

5. Moricz, F: Tauberian conditions under which statistical convergence follows from statistical summability $(C, 1)$. J. Math. Anal. Appl. 275, 277-287 (2002)

6. Mohiuddine, SA, Alotaibi, A: Korovkin second theorem via statistical summability (C, 1). J. Inequal. Appl. 2013, 149 (2013)

7. Mursaleen, M, Alotaibi, A: Statistical summability and approximation by de la Vallée-Poussin mean. Appl. Math. Lett. 24, 320-324 (2011) (Erratum: Appl. Math. Lett. 25, 665 (2012))

8. Mursaleen, $\mathrm{M}$, Edely, OHH: On the invariant mean and statistical convergence. Appl. Math. Lett. 22, $1700-1704$ (2009)

9. Edely, $\mathrm{OHH}$, Mursaleen, M: On statistical A-summability. Math. Comput. Model. 49, 672-680 (2009)

10. Colak, R, Bektas, CA: $\lambda$-Statistical convergence of order $\alpha$. Acta Math. Sci. 31(3), 953-959 (2011)

11. Mohiuddine, SA, Alghamdi, MA: Statistical summability through a lacunary sequence in locally solid Riesz spaces. J. Inequal. Appl. 2012, 225 (2012)

12. Mohiuddine, SA, Alotaibi, A, Mursaleen, M: Statistical convergence through de la Vallée-Poussin mean in locally solid Riesz spaces. Adv. Differ. Equ. 2013, 66 (2013)

13. Mohiuddine, SA, Aiyub, M: Lacunary statistical convergence in random 2-normed spaces. Appl. Math. Inf. Sci. 6(3), 581-585 (2012)

14. Mohiuddine, SA, Alotaibi, A, Alsulami, SM: Ideal convergence of double sequences in random 2-normed spaces. Adv. Differ. Equ. 2012, 149 (2012)

15. Belen, C, Mohiuddine, SA: Generalized weighted statistical convergence and application. Appl. Math. Comput. 219 9821-9826 (2013)

16. Malkowsky, E, Mursaleen, M, Suantai, S: The dual spaces of sets of difference sequences of order $m$ and matrix transformations. Acta Math. Sin. Engl. Ser. 23, 521-532 (2007)

17. Savaş, E, Kiliçman, A: A note on some strongly sequence spaces. Abstr. Appl. Anal. 2011, Article ID 598393 (2011)

18. Connor, JS: The statistical and strong p-Cesàro convergence of sequences. Analysis 8, 47-63 (1988)

doi:10.1186/1687-1847-2013-212

Cite this article as: Alotaibi and Mursaleen: Generalized statistical convergence of difference sequences. Advances in Difference Equations 2013 2013:212 